\title{
Circuit Model of Carbon-Nanotube Inks for Microelectronic and Microwave Tunable Devices
}

\author{
Rosa De Paolis ${ }^{1}$, Sébastien Pacchini ${ }^{2,3}$, Fabio Coccetti ${ }^{2,3}$, Giuseppina Monti ${ }^{1}$, Luciano Tarricone ${ }^{1}$, \\ Manos M. Tentzeris ${ }^{4}$, and Robert Plana ${ }^{2,3}$ \\ ${ }^{1}$ University of Salento, Lecce, 73100 , Italy \\ ${ }^{2}$ CNRS; LAAS, 7 avenue du colonel Roche, F-31077 Toulouse, France \\ ${ }^{3}$ Université de Toulouse; UPS, INSA, INP, ISAE; LAAS, F-31077 Toulouse, France \\ ${ }^{4}$ GEDC, School of ECE, Georgia Institute of Technology, Atlanta, GA 30332, U.S.A.
}

\begin{abstract}
This paper proposes an electrical model of carbon-nanotube (CNT) networks, that can be patterned using inkjet transfer printing. The Double-Walled carbon Nanotubes (DWNTs) "inkjet"-able suspension was deposited in gaps of variable lengths cut out of the central line of the coplanar waveguide test structure. The proposed model was validated through measurements of the line input impedance and of the scattering parameters. The obtained results demonstrate that, by acting on the gap size and on the number of CNT ink layers, both the DC resistance and the resonance frequency of the test structure can be selected ad hoc (from tens of $\mathrm{k} \Omega$ to tens of $\Omega$ and from few $\mathrm{MHz}$ to tens of $\mathrm{MHz}$, respectively). Therefore, by exploiting the suggested equivalent circuit model, the CNT ink deposition process can be considered as a promising candidate for the design of microelectronic and microwave devices with customized behavior (i.e., variable resistors, matching load networks, filters, and resonators).
\end{abstract}

Index Terms - carbon nanotubes, inkjet printing, nanotechnology, printed resonators, tunable circuits.

\section{INTRODUCTION}

Carbon Nanotubes (CNTs) are attractive nanoscale materials which have been gaining great interest both in the industrial and the academic world. Their unique mechanical electrical and thermal properties make them promising building blocks for a large variety of applications in microelectronics and in nanotechnologies. In particular, CNTs are expected to address effectively numerous common electronic system challenges, such as tunability, miniaturization, and cost reduction.

It has been recently demonstrated that CNTs were successfully deposited using an inkjet printing process [1], thus enabling their use in the fabrication of various patterns and electronic devices. In particular, Radio Frequency (RF) circuits, that include resonators, antennas, and wireless identification tags (RFID), were fabricated and tested [2]. Furthermore, the possibility to print high-frequency tunable circuits on paper (one of the cheapest existing materials) with CNTs ink has been demonstrated as well $[3,4,5]$.

Up to now only few preliminary studies have been reported on CNTs-based inkjet printing modeling and characterization [6]. On the other hand, the electrical resonance behavior of CNT-based networks is reported in [7], where a miniaturized
RF resonator made of CNTs has been fabricated and tested up to $400 \mathrm{MHz}$.

In this paper, an electrical circuit model of CNT-based networks, that are patterned using an inkjetable DoubleWalled carbon Nanotubes (DWNTs) deposition method, is proposed. Frequency measurements of the line input impedance up to $100 \mathrm{MHz}$ and of the scattering parameters up to $5 \mathrm{GHz}$ have been performed on several samples, showing that the magnitude of the line input impedance has a constant value up to few $\mathrm{MHz}$ and a resonant behavior around few tens of MHz. In addition, they confirm that the test structure behavior can be controlled by the gap size and by the number of deposited CNT ink layers. More specifically, the DC resistance can vary from tens of $\mathrm{k} \Omega$ to tens of $\Omega$, while the resonance frequency can vary from few $\mathrm{MHz}$ to tens of $\mathrm{MHz}$, with a potential tunability factor of about $200 \%$ for the resistance values and of about $65 \%$ for the resonance frequencies values. The comparison between measurements and simulation results will be presented and discussed.

Therefore, by means of the suggested equivalent circuit model, the properties of CNT-based printed circuits, can be efficiently explored, in terms of both resistance and resonance frequency, to develop a variety of microelectronic and microwave devices, such as sensors, resonators, resistors, and matching load networks.

\section{TEST StRUCtURE FABRICATION AND CNTS DEPOSITION PROCESS}

In order to investigate the electrical properties of the CNT ink, a test vehicle structure consisting of a coplanar waveguide $(\mathrm{CPW})$ on silicon substrate with a gap of variable length on the central line has been considered (Fig. 1a). A suspension made of $100 \mathrm{mg} / \mathrm{L}$ DWNTs, and mixed in ethylene-glycol, was used as the inkjetable material. The DWNTs are synthesized by the catalytic chemical vapor deposition (CCVD) method and undergo a thermal oxidation treatment in order to be functionalized, thus to form a stable suspension. The patterning on the CPW gap was finally performed by inkjet printing at room temperature [3] followed by a $100^{\circ} \mathrm{C}$ drying. A good contact between electrodes and 
DWNTs is obtained verifying the good homogeneity of the DWNTs networks.

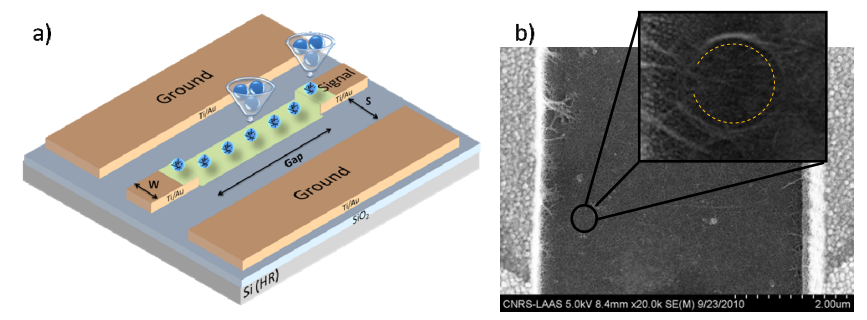

Fig.1. a) Coplanar waveguide structure with a gap of variable length on the central line, b) SEM pictures of the CNT ink deposition on a $6 \mu \mathrm{m}$ gap. Yellow dotted lines show the shape of the CNTs within the network.

CNT-based ink drops fill the variable-length gap. Considering that the drop diameter is around $50 \mu \mathrm{m}$, depending on the gap size, one or more adjacent drops can be needed. In order to investigate the effects on the behavior of the network of printed DWNTs, after the first print step, the pattern can be overwritten by the repetition of the filling process, i.e. by deposition of other layers of overlapping drops. Furthermore, it has been noticed that, by increasing the number of prints, the uniformity of the DWNTs was greatly improved over the whole area, giving rise to tangled randomly oriented networks of nanotubes (Fig. 1b).

\section{STRUCTURE DESCRIPTION AND EQUIVALENT CIRCUIT MODEL}

In order to investigate the dependence on the gap length and on the number of overwrites, measurements of the line input impedance value have been performed on several samples up to $100 \mathrm{MHz}$. The chosen parameters for the experimental parametrical investigation are the gap lengths $(6 \mu \mathrm{m}, 10 \mu \mathrm{m})$ and the number of consecutive overwrites $(1,2,4,5)$.

Measurements show that the magnitude of the line input impedance has a constant value up to about $10 \mathrm{MHz}$ and exhibits a resonant behavior around few tens of $\mathrm{MHz}$. As for its dependence on the gap and on the overwrites, it has been observed that: when the number of imprints increases, the curve of the impedance magnitude decreases and the resonance frequency becomes higher; whereas, when the gap length increases, the curve of the impedance magnitude increases and the resonance frequency becomes lower $[1,3,8]$.

In order to understand and exploit this technology flexibility, in this work an equivalent circuit model able to describe the measured behavior of the CNT ink is proposed. In our opinion the tangled randomly oriented networks of nanotubes can be seen as a cluster of randomly oriented ring resonators. It follows that the CNTs system can be modeled by a periodic network with unit cells consisting of a capacitance in parallel with the series of a resistance and an inductance (Fig. 2). Every unit cell represents a CNT or a portion of CNTs.

The existence of a large kinetic inductance has been theoretically predicted [9] and experimentally verified [10] for individual and bundled aligned Single-Walled carbon Nanotubes (SWNTs). In our case, the strong inductive behavior can be also explained by the tangled randomly oriented nanotubes, which can be considered as tiny coils as reported in [1].

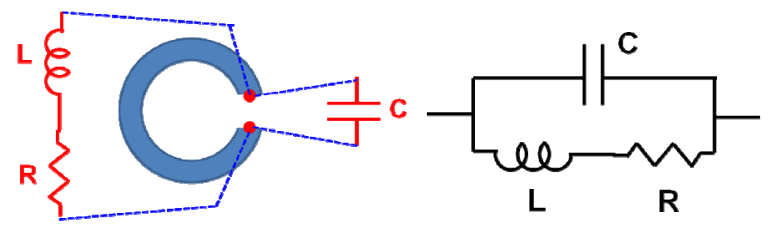

Fig.2. Unit cell of the equivalent circuit.

The tunneling effect between neighboring CNTs also has to be taken into account. In fact it has been demonstrated that it strongly affects the resistance and the electrical conductivity in randomly distributed CNTs, especially when the CNT loading is close to the percolation threshold [11]. Furthermore an electrical energy storage phenomenon in closely spaced layers, very similar to the one that takes place in microsupercapacitors based on onion-like carbon [12], can further justify the high capacitive behavior.

The impedance of the line up to about $10 \mathrm{MHz}$ is predominantly controllable by changing the resistance value $R$; whereas, at the resonance point and in the higher frequency region, the dominant contribution can be associated to inductive and capacitive effects, i.e. to $L$ and $C$ values.

By increasing the gap length and the number of overwrites, the amount of adjacent and overlapping CNTs increases. As a consequence, the unit cell will be replicated in series and in parallel branches. Thus, several coupling effects, both capacitive and inductive, have to be taken into account. In order to deal with a simple circuit model, the coupling between inductors and between parallel branches have been considered as the most prominent effects: according to measurement results, they can be modeled, respectively, by means of a coupling coefficient $k$ and by a capacitance $C_{c}$ and a resistance $R_{c}$ in shunt configuration, as shown in Fig. 3.

Furthermore, a contact resistance, modeling the connection points between the metal (electroplated gold) of the CPW line and the CNT network, and also a parasitic capacitance between the two open ends of the interrupted CPW central line (non-filled gap) have been considered and placed in the equivalent circuit network.

Considering the coupling of RF energy and mechanically vibrating CNTs, as reported for example in [13], we propose a circuit able to describe the CNTs network general behavior, without focalizing on any specific resonance contribution. 

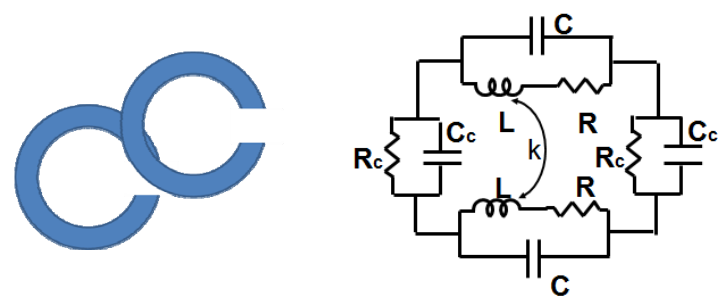

Fig.3. Two parallel unit cells with coupling effects.

\section{SIMULATIONS AND RESULTS}

The here proposed equivalent circuit model has been optimized and then verified by measurements performed on the CPW structure with a variable gap of $6 \mu \mathrm{m}$ and $10 \mu \mathrm{m}$ filled by 1 to 5 overwritten depositions of CNT ink. This choice has been made in order to carry out the analysis in the most repeatable sample conditions, obtained by single drop deposition and avoiding irregular capillarity phenomena taking place on smaller gaps $(<5 \mu \mathrm{m})$.

The chosen modeling procedure can be summarized as follows:

1) every unit cell represents the CNTs clustered in a $2 \mu \mathrm{m}$ length line with a single deposition layer;

2) every parallel branch is a different deposition layer;

3) all the deposition layers have been modeled by the same parameters, except the first and the last ones.

The circuit lumped elements have been optimized in order to obtain the same impedance behavior achieved by measurements. The parameter values extracted are summarized in Table I. Furthermore, the estimated contact resistance is around few $\mathrm{k} \Omega$. The parasitic capacitance between the two open ends of the interrupted CPW central line has been modeled by fitting measurements and full-wave simulations performed on the CPW structure without any CNT deposition: the extracted value is in the range of tens of $\mathrm{fF}$ for all the considered gaps. As for the coupling coefficient $k$, it does not affect strongly the overall behavior of the circuit, and its role should be further investigated.

The comparisons between simulation results performed on the proposed equivalent circuital model and measurements are shown in Fig. 4 and 5.

Simulation results display an impedance behavior very similar to the one provided by measurements. In particular, for a fixed gap length, as the number of overwrites is increased the impedance decreases and the resonance peak shifts toward higher frequencies (Fig. 4); for a fixed number of overwrites, as the gap length is increased the impedance increases and the resonance peak shifts toward lower frequencies (Fig. 5).

Afterwards measurements of scattering parameters up to 5 $\mathrm{GHz}$ have been compared with results obtained by full-wave and circuital simulations modeling the CPW test structures where the CNTs network is replaced by the equivalent circuit described so far.
TABLE I

SUMMARY OF CiRCUITAL PARAMETERS

\begin{tabular}{|c|c|c|c|}
\hline Circuital & \multicolumn{3}{|c|}{ Deposition layer } \\
Component & First & Intermediate & Last \\
\hline $\mathrm{C}[\mathrm{pF}]$ & 6.8 & 3.28 & 6 \\
\hline $\mathrm{L}[\mathrm{nH}]$ & 3800 & 109 & 1500 \\
\hline $\mathrm{R}[\mathrm{Ohm}]$ & 300 & 313 & 600 \\
\hline $\mathrm{R}_{\mathrm{c}}[\mathrm{Ohm}]$ & & 8.5 & 37.5 \\
\hline $\mathrm{C}_{\mathrm{c}}[\mathrm{pF}]$ & & 8.5 & 0.98 \\
\hline
\end{tabular}

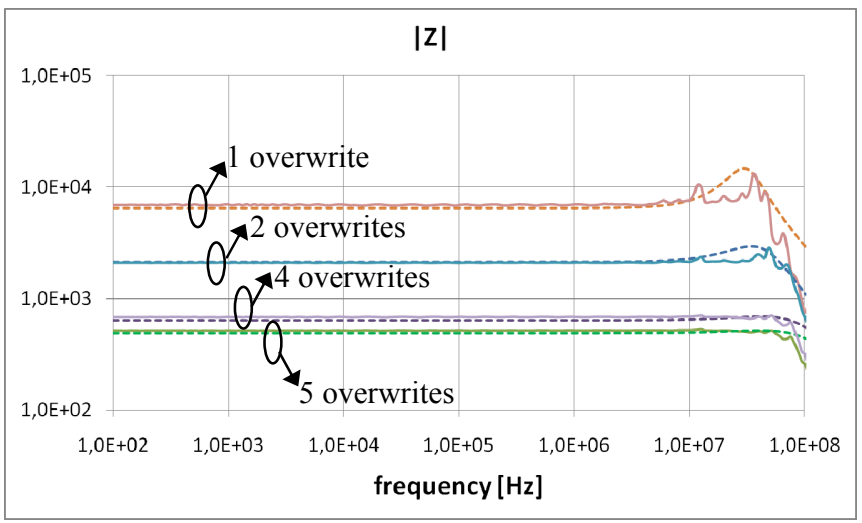

Fig.4. Input impedance magnitude for a fixed gap length of $10 \mu \mathrm{m}$ for a different number of overwrites: simulations performed on the equivalent circuital model (dotted lines) and measurements up to $100 \mathrm{MHz}$ (solid lines).

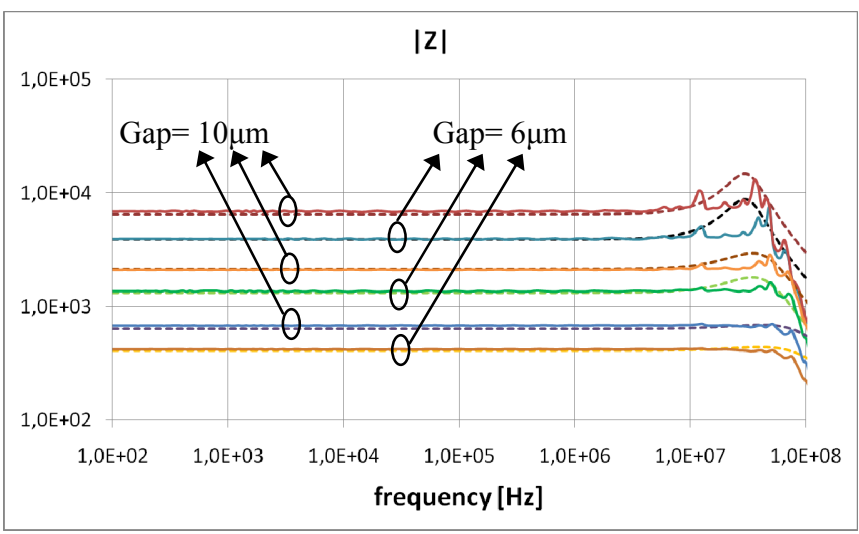

Fig.5. Input impedance magnitude for a gap length of $6 \mu \mathrm{m}$ and of $10 \mu \mathrm{m}$ : simulations performed on the equivalent circuital model (dotted lines) and measurements up to $100 \mathrm{MHz}$ (solid lines). Starting from above, every pair of curves corresponds to 1,2 , and 4 overwrites respectively.

An example of the comparison between measured and simulated results has been reported in Fig. 6. Especially at high frequencies a good matching can be noticed validating the proposed electrical modeling. One reason behind the mismatch, especially at very low frequencies, could be a frequency-dependent behavior of capacitive and inductive 
elements, as explained in [14]. Furthermore it can be noticed that by acting on the number of overwrites the S-parameters can be controlled in order to provide good input matching and transmission coefficient from tens of $\mathrm{k} \Omega$ to tens of $\Omega$.

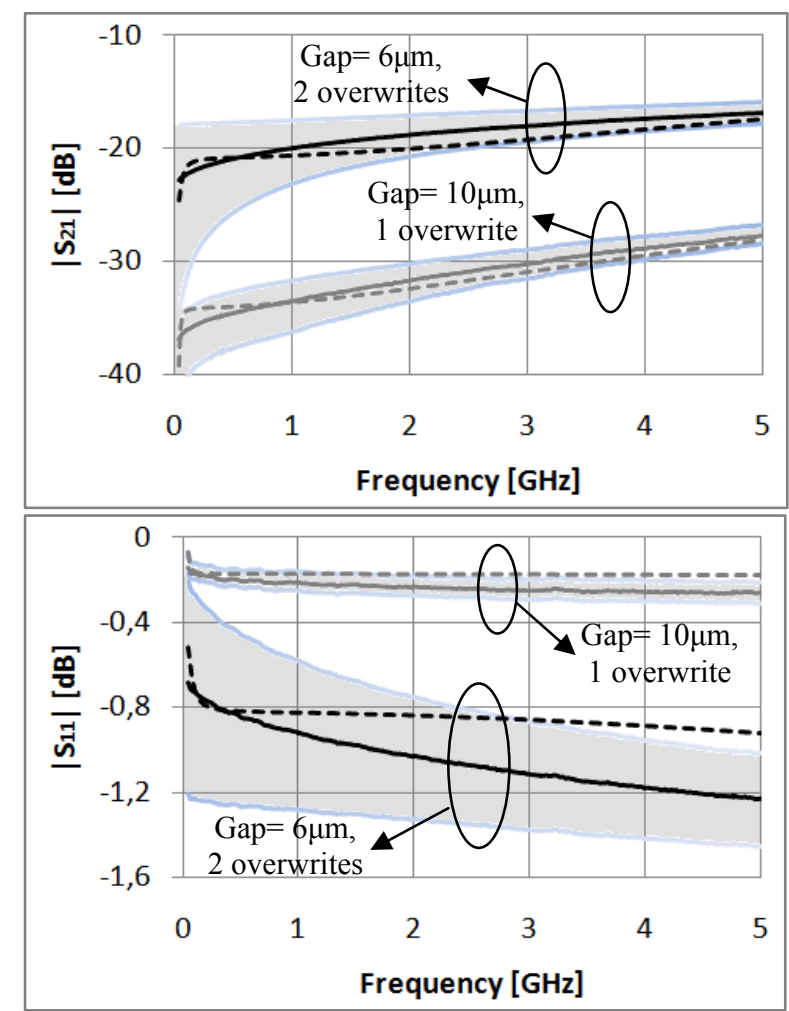

Fig.6. Scattering parameters: comparison between simulations (dotted lines) and measurements (shaded area for measurements performed on several samples and solid lines for their average).

\section{CONCLUSION}

In this paper an electrical model of carbon nanotubes network patterned using inkjet transfer printing is proposed. In order to demonstrate the validity of the suggested model frequency measurements of the line input impedance up to $100 \mathrm{MHz}$ and of scattering parameters up to $5 \mathrm{GHz}$ have been performed on several samples and compared with simulations. Furthermore the test structure exhibits a behavior that can be arbitrarily adjusted by acting on the gap size and on the number of CNT ink layers. Therefore, by exploiting the suggested equivalent circuit model, the CNT ink deposition process can be considered as a very promising candidate for the design of microelectronic and microwave devices with customized properties, such as sensors, resonators, resistors, and matching load networks.

\section{ACKNOWLEDGEMENT}

This work has been partially supported by the French National Agency (ANR) in the frame of its technological Research NANO-INNOV/RT program (NANOCOMM, project $\mathrm{N}^{\circ}$.ANR-09-NIRT-004) and the region Midi-Pyrénées. Moreover, we thank Norbert Fabre, the TEAM group of LAAS-CNRS, and Dr. Emmanuel Flahaut from CIRIMATCNRS for their contribution.

\section{REFERENCES}

[1] K. Kordás, T. Mustonen, T. Tóth, H. Jantunn, M. Lajunen, C. Soldano, S. Talapatra, S. Kar, R. Vajtai, and P.M. Ajayan, "Inkjet printing of electrically conductive patterns of carbon nanotubes," Small, vol. 2, no. 8-9, pp. 1021-1025, August 2006.

[2] L. Yang, A. Rida, R. Vyas, and M.M. Tentzeris, "RFID tag and RF structures on paper substrates using inkjet-printing technology," IEEE Trans. on Microwave Theory \& Tech., vol. 55, no.12, pp. 2894-2901, December 2007.

[3] S. Pacchini, V. Conedera, F. Mesnilgrente, N. Fabre, E. Flahaut, F. Coccetti, M. Dragoman, R. Plana, "Tunability of carbon nanotube resistance deposited by inkjet printing at low temperature," Mat. Res. Soc. Symp. Proc., vol. 1258, 2010.

[4] M. Dragoman, E. Flahaut, D. Dragoman, M. Al Ahmad, R. Plana, "Writing simple RF electronic devices on paper with carbon nanotube ink," Nanotechnology, vol. 20, no. 37, 2009.

[5] L. Yang, R. Zhang, D. Staiculescu, C.P. Wong, and M.M. Tentzeris, "A novel conformal RFID-enabled module utilizing inkjet-printed antennas and carbon nanotubes for gas-detection applications," IEEE Antennas and Wireless Propagation Letters, vol. 8, pp. 653-656, June 2009.

[6] S. Gagliardi, L. Giorgi, R. Giorgi, N. Lisi, Th. Dikonimos Makris, E. Salernitano, A. Rufoloni, "Impedance analysis of nanocarbon DSSC electrode," Superlattices \& Microstructures, vol. 46, no. 1-2, pp. 205-208, July-August 2009.

[7] M. A. El Sabbagh, and S. M. El-Ghazaly, "Miniaturized carbon nanotube-based RF resonator," IEEE MTT-S Int. Microwave Symp. Dig., pp.829-832, June 2009.

[8] J.-W. Song, J. Kim, Y.-H. Kim, Y.-H. Yoon, B.-S. Choi, J.-H. Kim, C.-S. Han, "Inkjet printing of single walled carbon nanotubes and electrical characterization of line pattern," Nanotechnology 19, pp. 095702, 2008.

[9] P. J. Burke, "Lüttinger liquid theory as a model of the gigahertz electrical properties of carbon nanotubes," IEEE Trans. on Nanotechnology, vol. 1, no. 3, pp.129-144, September 2002.

[10] J. J. Plombon, K. P. O'Brien, F. Gstrein, V. M. Dubin, and Y. Jiao, "High-frequency electrical properties of individual and bundled carbon nanotubes," Appl. Phys. Lett., vol. 90, no. 6, p. 063 106, February 2007.

[11] N. Hua, Y. Karub, C. Yan, Z. Masuda, H. Fukunaga, "Tunneling effect in a polymer/carbon nanotube nanocomposite strain sensor," Acta Materialia, vol. 56, no. 13, pp. 2929-2936, August 2008.

[12] D. Pech, M. Brunet, H. Durou, P. Huang, V. Mochalin, Y. Gogotsi, P-L. Taberna, and P. Simon, "Ultrahigh-power micrometre-sized supercapacitors based on onion-like carbon," Nature Nanotechnology, vol. 5, pp. 651-654, August 2010.

[13] J.F. Davis, M. Bronikowski, D. Choi, L. Epp, M. Hoenk,D. Hoppe, B. Kowalczyk, F. Noca, E. Wong, B. Hunt, B. Chang, M. Jouzi, M.Tzolov, A. Yin, J. Xu, J.D. Adam, R.M. Young, J. Adams, B. Rogers, "High-Q mechanical resonator arrays based on carbon nanotubes," IEEE Conference on Nanotechnology, vol. 2, pp. 635-638, August 2003.

[14] S.C. Jun, J.H. Choi, S. N. Cha, C. W. Baik, S. Lee, H. Jin Kim, J. Hone, and J. M. Kim, "Radio-frequency transmission characteristics of a multi-walled carbon nanotube," Nanotechnology, vol. 18, no. 15, p. 255701, June 2007. 